\title{
PROFILE OF LOCOREGIONAL RECURRENCE AND DISTANT METASTASIS OF BREAST CANCER IN BRAZILIAN WOMEN
}

\author{
Leonardo R. Soares ${ }^{1}$, Magno B. Cirqueira', Marise A. R. Moreira², Carolina R. Mendonça', Ruffo Freitas-Júnior ${ }^{1,3}$ \\ ${ }^{1}$ Health Sciences Program, Universidade Federal de Goiás - Goiânia (GO), Brazil. \\ ²Department of Pathological Anatomy, Hospital das Clínicas, Universidade Federal de Goiás - Goiânia (GO), Brazil. \\ ${ }^{3}$ Mastology Program, Universidade Federal de Goiás; Hospital Araújo Jorge, Associação de Combate ao Câncer em Goiás - \\ Goiânia (GO), Brazil.
}

Objective: To evaluate the locoregional recurrence and distance metastasis profile in women with breast cancer. Methods: Retrospective, cross-sectional study conducted at a tertiary hospital. Data from medical records of women diagnosed between 2003 and 2007 with infiltrating ductal carcinoma of the breast were analyzed; all cases with information available were included. Data collection was performed until October 2018. Variables related to local recurrence or distant metastases from breast cancer, as well as mortality rate, were reviewed. Statistical analyses were performed in the statistical package Stata, version 13.0. Results: In the data analysis, 195 patients were included, with a mean follow-up of 109 months. The mean age at diagnosis was $50.3 \pm 0.89$ years. Immunohistochemistry demonstrated $77.4 \%$ luminal tumors, $13.3 \%$ HER2 overexpression and 5.1\% triple-negative tumors. The overall recurrence rate was 44.6\% (87/195), with 9.2\% locoregional recurrence, $39.5 \%$ at distance, and 5.6\% both. Among the cases of distant metastases, $25.6 \%$ occurred in bones, $21 \%$ in the lungs, $14.4 \%$ in the liver, $8.2 \%$ in the brain, $1.5 \%$ in the peritoneum, and $0.5 \%$ in the adrenal. Relapse-free survival was 43 months and for distant metastases 48 months. The overall mortality rate was $54.36 \%(106 / 195)$ and the mean time to death was 69 months. Conclusion: There was a considerable rate of recurrence, mainly at a distance, with a predominance of bone, pulmonary, and hepatic metastases. The high mortality rate observed in the present study may be due to limitations in the treatment of the disease, advanced diagnosis, and factors related to tumor biology. 